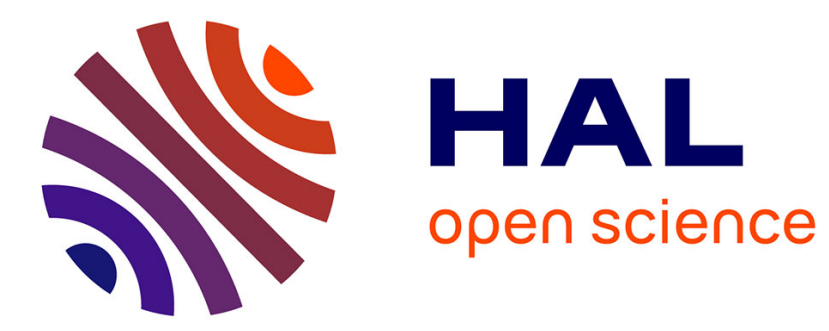

\title{
Sur l'attraction de deux plateaux séparés par un diélectrique
}

Julien Lefèvre

\section{To cite this version:}

Julien Lefèvre. Sur l'attraction de deux plateaux séparés par un diélectrique. J. Phys. Theor. Appl., 1892, 1 (1), pp.243-245. 10.1051/jphystap:018920010024300 . jpa-00239613

\section{HAL Id: jpa-00239613 https://hal.science/jpa-00239613}

Submitted on 1 Jan 1892

HAL is a multi-disciplinary open access archive for the deposit and dissemination of scientific research documents, whether they are published or not. The documents may come from teaching and research institutions in France or abroad, or from public or private research centers.
L'archive ouverte pluridisciplinaire HAL, est destinée au dépôt et à la diffusion de documents scientifiques de niveau recherche, publiés ou non, émanant des établissements d'enseignement et de recherche français ou étrangers, des laboratoires publics ou privés. 
LEFÈVRE. - ATTRAGTION A TRAVERS UN DIÉLEGTRIQUE. $2 q^{3}$

\section{SUR L'ATTRaction DE DEUX PLATEAUX SÉParís PAR UN DIÉlEGTRIQUe;}

Par M. Julien Lefèvre.

J'ai mesuré l'attraction de deux plateaux électrisés, séparés par un diélectrique non en contact intime avec eux, et vérifié qu'elle est représentée par la formule suivante

$$
\frac{\mathbf{F}}{\overline{\mathbf{F}}^{\prime}}=\left(\frac{e+e^{\prime}}{\frac{e}{k}+e^{\prime}}\right)^{2},
$$

en appelant $\mathrm{F}^{\prime}$ l'attraction des deux plateaux à la distance $e+e^{\prime}$ dans l'air; F l'attraction à la même distance lorsqu'on a placé entre les plateaux une lame isolante à faces parallèles d'épaisseur $e$; $e^{\prime}$ représente donc la somme des épaisseurs d'air situées de part et d'autre de cette lame; $k$ est la constante diélectrique de la plaque.

Je me sers de deux plateaux horizontaux et d'une balance de précision dont le fléau porte, à une extrémité, un plateau ordinaire pour recevoir des poids, à l'autre, le plateau mobile de l'appareil électrique, qui a $2_{2}{ }^{\mathrm{cm}}$ de diamètre et est suspendu au fléau par une tige isolante. Ce plateau est entouré d'un anneau de garde, muni d'une sorte de couvercle, qui est seulement percé d'un trou pour laisser passer la tige isolante.

Le plateau fixe, qui a $9^{\mathrm{cm}}$ de diamètre, est placé au-dessous du premier et porté par un support isolant à vis calantes, qui permet de faire varier la distance $e+e^{\prime}$. Ce support est traversé par trois tiges isolantes qui soutiennent le diélectrique. De part et d'autre de celui-ci, on laisse des épaisseurs d'air aussi faibles que possible; il faut cependanı laisser au plateau mobile la possibilité d'accomplir de petites oscillations.

La source d'électricité est une bobine de Ruhmkorff, actionnée par 6 éléments Bunsen; l'un des pôles de la bobine communique avec le plateau fixe et avec l'armature intérieure d'une jarre dont la capacité est, suivant les cas, zo à i 5o fois plus grande que celle du condensateur formé par les plateaux. L'autre pôle, l'armature extérieure de la jarre, le plateau mobile et le fléau de la balance sont reliés au sol. 
Tout l'appareil, sauf le plateau qui reçoit les poids, est placé dans une cage dont l'air est desséché.

Les plaques de soufre et de paraffine sont obtenues par fusion, dans un moule dont le fond est rendu horizontal par un niveau à bulle d'air. L'épaisseur des lames solides est mesurée avec une vis à deux pointes et un cathétomètre. La distance $e+e^{\prime}$ des deux plateaux est aussi mesurée au cathétomètre.

Le diélectrique est placé dans la cage quelques jours avant l'expérience, pour lui laisser perdre toute trace d'électricité.

La balance contenant une tare un peu trop faible et toutes les pièces communiquant avec le sol, j'achève d'établir l'équilibre avec un poids $p$. J'électrise ensuite le plateau fixe et j'établis de nouveau l'équilibre par un poids $P$. Je remets ce plateau en communication avec le sol et j'équilibre encore par un poids $p^{\prime}$ :

$$
\mathbf{F}=\mathrm{P}-\frac{p+p^{\prime}}{2}
$$

L'attraction $\mathbf{F}^{\prime}$ est déterminée de même par trois opérations.

J'opère de même pour les liquidés, mais en mesurant d'abord $\mathrm{F}^{\prime}$, puis $\mathbf{F}$, afin d'éviter l'évaporation. L'épaisseur d'air $e^{\prime}$ subit dans ce cas une petite correction qui consiste à remplacer l'épaisseur de la lame de verre formant le fond de la cuve qui reçoit le liquide par l'épaisseur d'air équivalente.

Dans chaque expérience, je calcule le rapport $\frac{F}{F^{\prime}}$. puis le rapport corrigé

$$
\mathrm{R}=\frac{\mathrm{F}}{\mathrm{F}^{\prime}}\left(\begin{array}{c}
e+k e^{\prime} \\
e+e^{\prime}
\end{array}\right)^{2}
$$

D’après (I), on doit avoir

$$
\sqrt{\mathrm{R}}=k \text {. }
$$

Le Tableau suivant, dans lequel $e$ et $e^{\prime}$ sont exprimés en centimètres, $F$ ct $F^{\prime}$ en milligrammes, montre que les valeurs de $\sqrt{\mathbf{R}}$ concordent avec les valeurs de $k$ que j'ai obtenues au moyen de la balance de Coulomb (').

( $\left.{ }^{1}\right)$ Comptes rendus des séances de l'Académie des Sciences, 16 novembre 189 . 
La formule (I) est donc exacte et, en particulier, dans le cas où $e^{\prime}$ devient négligeable, on a

$$
\frac{\mathrm{F}}{\mathrm{F}^{\prime}}=k^{2}
$$

Diélectriques. $\quad$ e. $\quad e^{\prime} . \quad \mathrm{F} . \quad \mathbf{F}^{\prime} \cdot \frac{\mathbf{F}}{\mathbf{F}^{\prime}} \cdot \quad$ R. $\quad \sqrt{\bar{R}} . \quad k$.

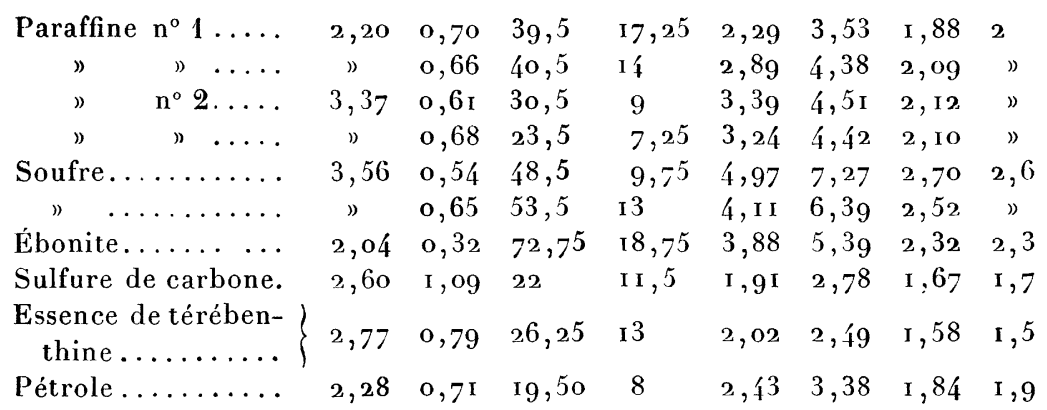

Je ferai remarquer, en terminant, que l'attraction de deux plaleaux se prête parfaitement à la mesure des constantes diélectriques; c'est une méthode simple, rapide et n'exigeant qu'une balance de précision; elle est préférable à tous les procédés actuellement en usage. 